\title{
Régimen natural de caudales del río Ayuquila-Armería en el occidente de México
}

\author{
Natural flow regime of the Ayuquila-Armeria River in western Mexico \\ Demetrio Meza Rodríguez ${ }^{1}$, Luis Manuel Martínez Rivera ${ }^{1+}$, Norman Mercado Silva ${ }^{2}$,
Diego García de Jalón Lastra ${ }^{3}$, Marta González del Tánago del Rio ${ }^{3}$,
Miguel Marchamalo Sacristán ${ }^{4}$ y Celia De la Mora Orozco
} ${ }^{1}$ Departamento de Ecología y Recursos Naturales, Universidad de Guadalajara. Independencia Nacional \#151. 48900 Autlán de Navarro, Jalisco, México.
${ }^{7}$ Autor responsable (lmartinez@cucsur.udg.mx)
${ }^{2}$ Centro de Investigación en Biodiversidad y Conservación, Universidad Autónoma del Estado de Morelos. Avenida Universidad 1001, Chamilpa. 62209
Cuernavaca, Morelos, México.
${ }^{3}$ Escuela de Ingenieros Forestales y del Medio Natural, Laboratorio de Hidrobiología, Universidad Politécnica de Madrid. Calle Ramiro de Maeztu 7. 28040
Madrid, España.
${ }^{4}$ Escuela Técnica Superior de Ingenieros de Caminos, Canales y Puertos, Laboratorio de Morfología del Terreno, Universidad Politécnica de Madrid. Calle Prof.
Aranguren 3. 28040 Madrid, España.
${ }^{5}$ Instituto Nacional de Investigaciones Forestales, Agrícolas y Pecuarias. Carretera Tepatitlán-Lagos de Moreno km 8. Col. Rancho Las Cruces. 47600 Tepatitlán
de Morelos, Jalisco, México.

\section{RESUMEN}

El caudal superficial a nivel cuenca, constituye hoy en día un proceso clave para los estudios hidrológicos de cualquier índole. El presente trabajo explora la reconstrucción del régimen natural de caudales de la cuenca del Río Ayuquila-Armería para el periodo 1963-1991, su importancia radica en que el método usado, puede ser extrapolado a otras cuencas en México y Latinoamérica, donde se carece de datos hidrométricos puntuales. El campo de interés está centrado en la técnica de reconstrucción del caudal natural basándose en las precipitaciones y las temperaturas, en situaciones donde se carece de información hidrológica puntual, utilizando el modelo determinístico "precipitaciónescurrimiento". Es un modelo que requiere de pocos parámetros para su implementación, usando los procesos climáticos y físicos en el ámbito espacial y temporal, con la utilización del Sistema de Información Geográfica (SIG). A partir de caudales calibrados en la misma área geográfica, es posible extrapolar el modelo de predicción a toda la cuenca. El resultado fue una base de datos de 29 años de régimen natural de caudales, con un caudal aproximado de $71.5\left(\mathrm{~m}^{3} \mathrm{~s}^{-1}\right)$, y con un volumen anual de $2254\left(\mathrm{hm}^{3}\right.$ año-1), donde se observaron las pautas estacionales y su fluctuación a lo largo del año. El uso de esta metodología servirá de apoyo para la nueva norma NMX-AA-159-SCFI-2012, donde el principio científico para la determinación de caudal ecológico es el paradigma del régimen hidrológico natural.

Cita recomendada:

Meza Rodríguez, D., L. M. Martínez Rivera, N. Mercado Silva, D. García de Jalón Lastra, M. González del Tánago del Rio, M. Marchamalo Sacristán y C. De la Mora Orozco. 2017. Régimen natural de caudales del río AyuquilaArmería en el occidente de México. Terra Latinoamericana 35: 203-217.
Palabras clave: precipitación-escurrimiento, modelo de simulación, caudal subterráneo, caudal superficial.

\section{SUMMARY}

Surface flow at the basin level is now a key process in hydrological studies of any kind. This research explores the reconstruction of the natural flow regime of the Ayuquila-Armería River basin for the period 1963-1991. The importance of reconstructing the flows in this study is that the designed method can be extrapolated to other basins in Mexico and Latin America where there are no specific hydrometric data. The field of study of interest is focused on the technique of reconstruction of natural flow based on precipitation and temperatures in situations where there is no specific hydrological information, using the deterministic model "precipitation-runoff". This model requires few parameters for its implementation. Using the Geographic Information Systems (GIS), climatic and physical processes in the space and time domain are defined. From calibrated flows in the same geographical area, it is possible to extrapolate the prediction model to the entire basin. The result was a 29 -year database of natural flow regime, which showed a flow rate of approximately $71.5\left(\mathrm{~m}^{3} \mathrm{~s}^{-1}\right)$ and an annual volume of $2254\left(\mathrm{hm}^{3}\right.$ year $\left.{ }^{-1}\right)$; seasonal patterns and fluctuations were observed throughout the year. The use of this methodology will support the new standard NMX-AA159-SCFI-2012, for which the scientific principle for 
determination of ecological flow is a paradigm of the natural hydrological regime.

Index words: precipitation-runoff, simulation model, subterranean flow, surface flow.

\section{INTRODUCCIÓN}

En la actualidad existen modelos de simulación continua del ciclo hidrológico a nivel cuenca, por ejemplo: Water Erosión Prediction Project (WEPP) y Soil and Water Assessment Tool (SWAT), sin embargo, aunque valiosos, requieren de una gran cantidad de información, de la cual muchas cuencas en México carecen, por lo que se reduce la eficiencia del modelo (Flores-López et al., 2003; Barrios y Urribarri, 2010).

En México actualmente, se establecen las especificaciones y los métodos para determinar escurrimiento de agua a través de la Norma Oficial Mexicana NOM-011-CNA-2015, el resultado es un volumen en $\mathrm{hm}^{3}$ año ${ }^{-1}$. Este resultado es deficiente para analizar la variabilidad intranual que se da a lo largo del río, y para los objetivos planteados en este estudio en la reconstrucción de un régimen natural de caudales a una escala mensual en $\mathrm{m}^{3} \mathrm{~s}^{-1}$.

A raíz de esta deficiencia metodológica y de información, se propone un método adaptado a la realidad mexicana para reconstruir el régimen natural de caudales en términos de su fluctuación intranual en $\mathrm{m}^{3} \mathrm{~s}^{-1}$, clasificándolo en superficial y subterráneo, con base en datos de precipitación $(\mathrm{mm})$ que son o están más ampliamente disponibles para muchas regiones del país. En este estudio se propone el modelo de Témez (1977), el cual es un modelo determinístico de paso mensual, ha sido utilizado ampliamente en España a través del SIMPA (Sistema Integrado de Modelación Precipitación Aportación), para poder llevar a cabo estimaciones de caudales con base en la precipitación en situaciones donde se carece de información hidrológica puntual (Estrela, 1992; Pizarro et al., 2005; Murillo y Navarro, 2011).

La cuenca del Río Ayuquila-Armería presenta procesos de alteración hidrológica, por este motivo es necesario conocer las condiciones naturales de distribución de los caudales dentro del río. Dado que la presa Tacotán se empezó a construir entre 1951-1958, el caudal del río, es un caudal alterado a la salida de la cuenca y es difícil conocer el régimen natural con la información hidrométrica existente. Por esta razón el modelo de Témez con base en la distribución mensual ofrece una alternativa metodológica. Este modelo trabaja con valores medios de las variables de precipitación y temperatura que están más disponibles para la cuenca. El objetivo de la reconstrucción de caudales, es caracterizar el régimen natural de caudales a una escala mensual, utilizando la distribución espacial de los factores que intervienen en el ciclo hidrológico, usando el Sistema de Información Geográfica (SIG). El SIG proporciona diversas funciones de análisis que permiten manipular las diferentes variables, utilizadas en los procesos de estimación de los parámetros hidrológicos de distribución temporal y espacial, planteadas por Témez. El objetivo de este trabajo fue analizar la metodología para la reconstrucción del régimen natural de caudales en condiciones de flujo alterado, que nos permitan entender los procesos de escurrimiento para facilitar la toma de decisiones para el mejor uso y aprovechamiento de los recursos hídricos de la cuenca.

\section{MATERIALES Y MÉTODOS}

\section{Área de Estudio}

La cuenca del Río Ayuquila-Armería se encuentra entre los estados de Jalisco y Colima en el occidente de la República Mexicana en las coordenadas geográficas $18^{\circ} 51^{\prime} 05^{\prime \prime}$ a $20^{\circ} 28^{\prime} 03^{\prime \prime} \mathrm{N}$ y $104^{\circ} 38^{\prime} 17^{\prime \prime}$, a $103^{\circ} 34^{\prime} 41^{\prime \prime} \mathrm{O}$ (Figura 1). Las corrientes principales son: el Río Ayuquila y Tuxcacuesco que nacen en la Sierra de Quila y la confluencia de estas forman la corriente del Río Armería. La cuenca drena una superficie aproximada de $9864 \mathrm{~km}^{2}$ con un trayecto de $321 \mathrm{~km}$ desde su nacimiento hasta su desembocadura en Boca de Pascuales en el estado de Colima. En la cuenca se identificaron trece unidades de suelos de la carta 1:250 000 del Instituto Nacional de Estadística y Geografía (INEGI), Serie II del año 2013 (INEGI, 2013). Destacan como abundantes en la superficie de la cuenca, los leptosoles con un $24 \%$ se encuentran en topográficas fuertemente disectada, los regosoles con $22 \%$ con materiales no consolidados, los phaeozem con $16 \%$ ricos en materia orgánica y los cambisoles con $16 \%$ con un horizonte subsuperficial incipiente (FAO, 2015). 


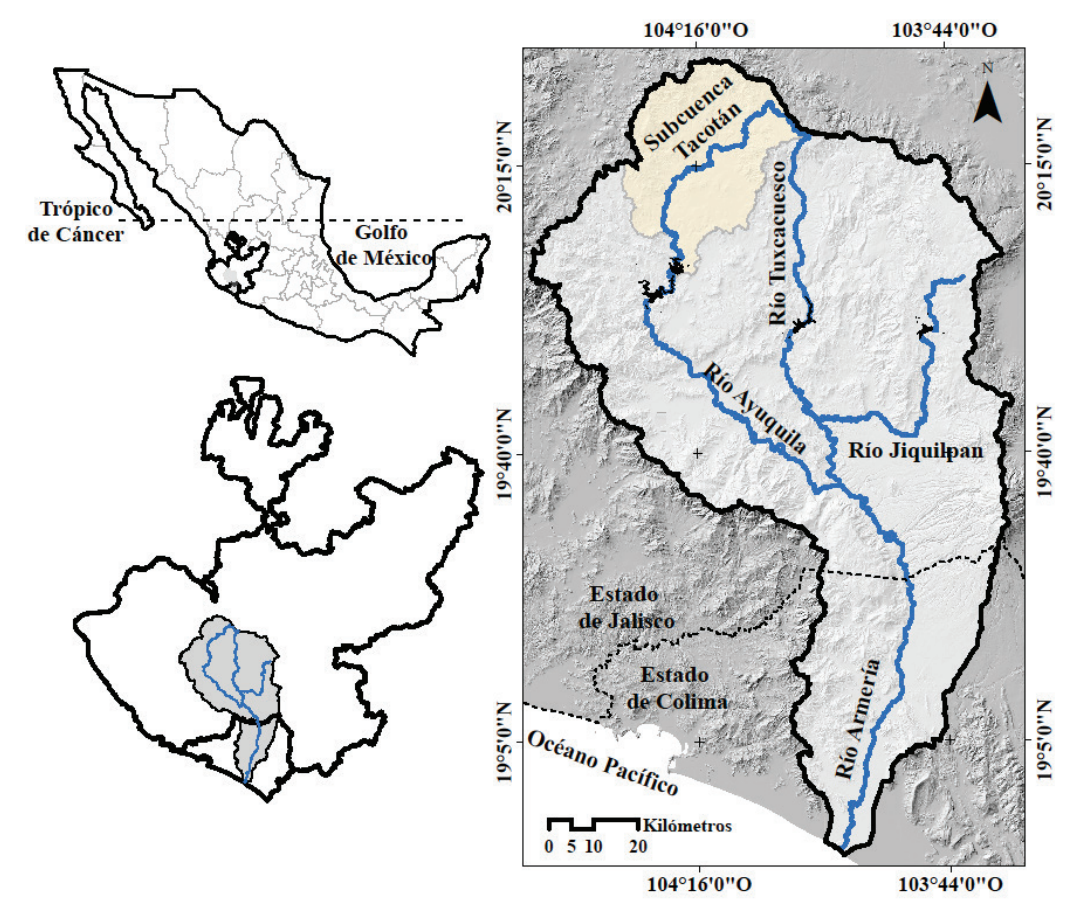

Figura 1. Localización de la cuenca del Río Ayuquila-Armería en el ámbito nacional.

\section{Modelo de Témez}

Pertenece al grupo de modelos agregados de simulación de cuencas, reproduce el ciclo hidrológico de una manera continua a lo largo del tiempo de una manera simple y conceptual (Estrela, 1992). Los datos de inicio en el modelo son los de precipitación (P), está, se fracciona en tres componentes, el primero, la evapotranspiración real (ER), resultado de la precipitación $(\mathrm{P})$ menos el excedente $(\mathrm{T})$, este resultado tiene lugar en la disponibilidad de agua en el suelo (X) y la reserva de agua en el suelo $(\mathrm{H})$ al siguiente mes; la disponibilidad de agua en el suelo (X) alimenta el cálculo para determinar evapotranspiración. El segundo denominado como excedente ( $\mathrm{T}$ ), es la suma del caudal superficial (e) y la infiltración (I) que tiene lugar a través del suelo para nutrir el almacenamiento en la capa inferior (fe), generando caudal lento o diferido (flujo base) (f). El tercero, el caudal total (At), es la suma del caudal superficial (e) y caudal base (f) (Figura 2).

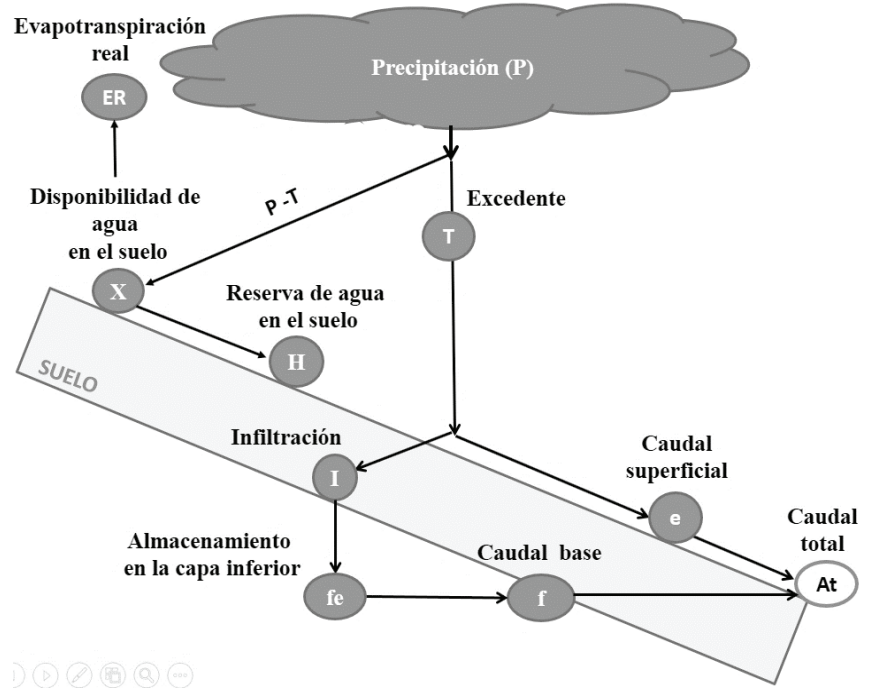

Figura 2. Descripción esquemática del modelo de Témez. 
Este modelo de índole general puede aplicar a cualquier intervalo de tiempo (hora, día, semana, mes, año), sin embargo, el intervalo temporal que más se usa es el mensual (Murillo y Navarro, 2011). En el Cuadro 1, se resumen las variables utilizadas por el modelo, sus unidades y las ecuaciones que rigen los diferentes subprocesos. Para mayor detalle del modelo ver Témez (1977).

\section{Subcuenca Tacotán}

Para el desarrollo de este estudio fue necesario utilizar los datos de la estación hidrométrica 16018 Tacotán localizada en la subcuenca del mismo nombre (Figura 1). En este punto se localiza la presa Tacotán en las coordenadas geográficas $20^{\circ} 02^{\prime} 07^{\prime}$ N N y $104^{\circ} 19^{\prime} 12 "$ O (Figura 3). Los datos fueron extraídos del Banco Nacional de Aguas Superficiales (BANDAS) de la Comisión Nacional de Agua (CONAGUA) (CONAGUA-SEMARNAT-IMTA, 2008), esta porción de la cuenca tiene información hidrométrica para un periodo de 36 años (1943-1978), en esta investigación se han considerado los datos de entrada de caudal a la presa como régimen natural para un periodo de 28 años (1951-1978), el estudio se dividió en dos etapas: una para calibrar y otra para verificar, utilizando los caudales calculados por el modelo y los observados por la estación hidrométrica. Validado el modelo mediante la aplicación de fórmulas matemáticas de Témez, se extendieron a toda la cuenca a partir de corrientes calibradas en la misma área geográfica, como resultado final es la reconstrucción del régimen natural de caudales de 1963-1991.

De las estaciones climáticas en la etapa de calibración, se utilizó la información de precipitación (mm) y temperatura $\left({ }^{\circ} \mathrm{C}\right)$ de seis estaciones (Figura 3 ) de 1943-1978. Para la simulación de toda la cuenca se usó la información de 58 estaciones climáticas para el periodo 1963-1991, la información proviene de la base de datos CLICOM del Servicio Meteorológico Nacional (SMN) (SMN, 2014).

El modelo de Témez consta de tres parámetros que tienen un significado físico: (1) coeficiente de escorrentía (Ce), (2) capacidad de campo $\left(\mathrm{H}_{\max }\right)$, y (3) coeficiente de infiltración $\left(\mathrm{C}_{\mathrm{i}}\right)$. Estos parámetros regulan el denominado umbral de escorrentía, capacidad máxima de infiltración y coeficiente de recesión de los acuíferos. Fueron obtenidos a partir de información fisiográfica de las cuencas. La información disponible en el estudio se integró en un Sistema de Información Geográfica (SIG) (Flores-López et al., 2003), estos distribuyen de manera espacial los parámetros físicos y los climáticos (precipitación y temperatura), dentro del ciclo hidrológico.

Para generar los diferentes mapas cartográficos de los parámetros expuestos anteriormente en la subcuenca Tacotán y cuenca Ayuquila-Armería, se utilizó la información cartográfica digital de INEGI, respecto a uso de suelo y vegetación de la Serie V del Inventario Nacional Forestal 2011-2013 (INEGI, 2013), y las capas temáticas edafológicas descritas anteriormente, empleando la información de la textura del suelo.

El Modelo Digital de Elevación (MDE), necesario para estimar la pendiente, se generó usando la información de 22 cartas topográficas digitales que forman la cuenca Ayuquila-Armería a escala 1:50000 (INEGI, 2015), usando la interpolación del modelo Kriging ordinario, se cuantificó la estructura espacial de los datos mediante el uso de variograma lineal, usando estadística, se asume que los datos más cercanos tienen mayor peso o influencia sobre la interpolación, disminuye conforme se aleja del punto de interés (Schloeder et al., 2001). Para estimar la pendiente, se obtuvo del MDE aplicando el método de Horn (1981), este método se utiliza para el cálculo del valor de los vecinos más cercanos y los vecinos diagonales, teniendo mayor peso los primeros. El valor es asignado al punto central, aunque su elevación no se utilice en el cálculo.

La distribución de la precipitación mensual se estimó a partir del método de interpolación de Kriging ordinario y para la temperatura mensual se realizó un gradiente alto térmico relacionando la temperatura con la altura de las estaciones meteorológicas utilizando el modelo de regresión obtenido y aplicándolo al MDE. Para el manejo de las cartas de INEGI y la generación digital cartográfica (sobreposición y álgebra de mapas) se utilizó el programa ArcGis 10.1.

\section{Coeficiente de Escorrentía (Ce)}

Para estimar este parámetro se clasificaron los suelos de la cuenca, en tres diferentes tipos de textura: A (suelos permeables); B (suelos medianamente permeables), y C (suelos casi impermeables), a partir de la información expuesta por Treviño et al. (2002) y Flores-López et al. (2003) definidos en el Cuadro 2. 
Cuadro 1. Parámetros propuestos por Témez (1977).

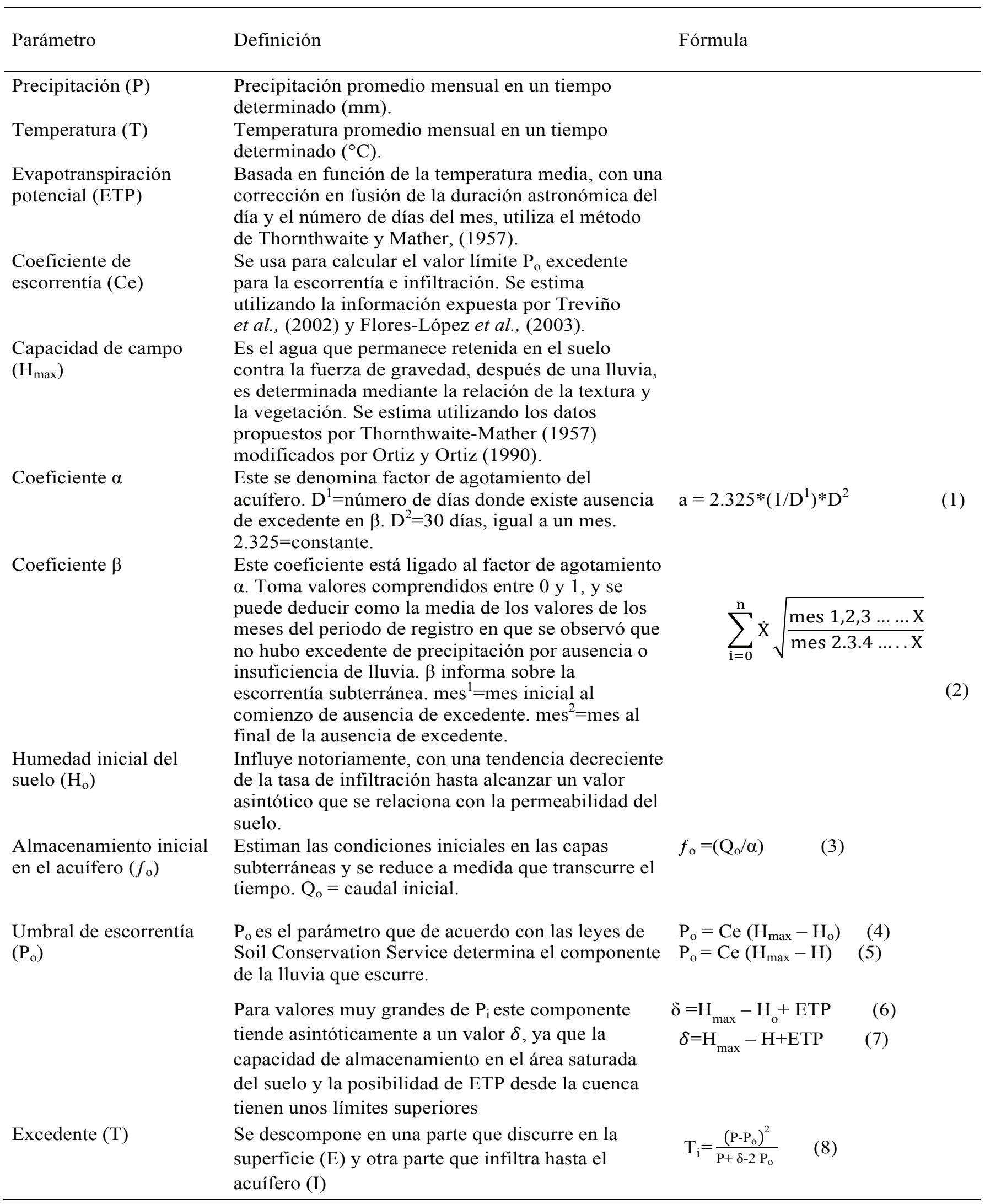


Cuadro 1 (continuación). Parámetros propuestos por Témez (1977).

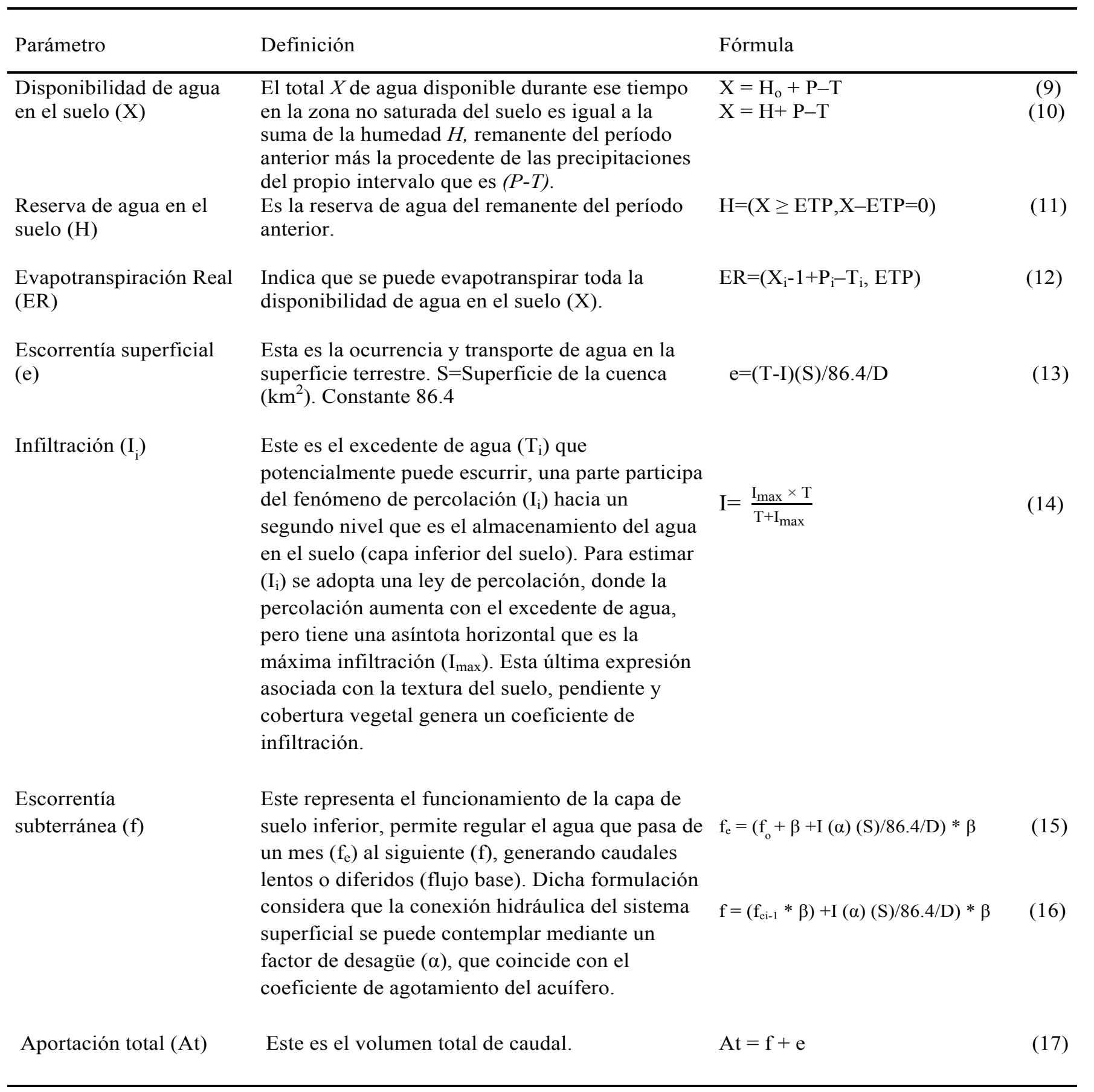

\section{Capacidad de Campo $\left(\mathrm{H}_{\max }\right)$}

La capacidad de campo fue determinada mediante la relación de la textura y la vegetación (Thornthwaite-Mather, 1957). Esta propuesta de Thornthwaite-Mather ha sido estudiada para determinar de manera indirecta la capacidad de retención de agua, relacionando valores a cada tipo de textura del suelo (Ortiz y Ortiz, 1990). El método propone relacionar el tipo de vegetación, con la textura del suelo presente en la cuenca. Se utilizaron los datos propuestos por ThornthwaiteMather (1957) modificados por Ortiz y Ortiz (1990), expuestos en el Cuadro 3. 


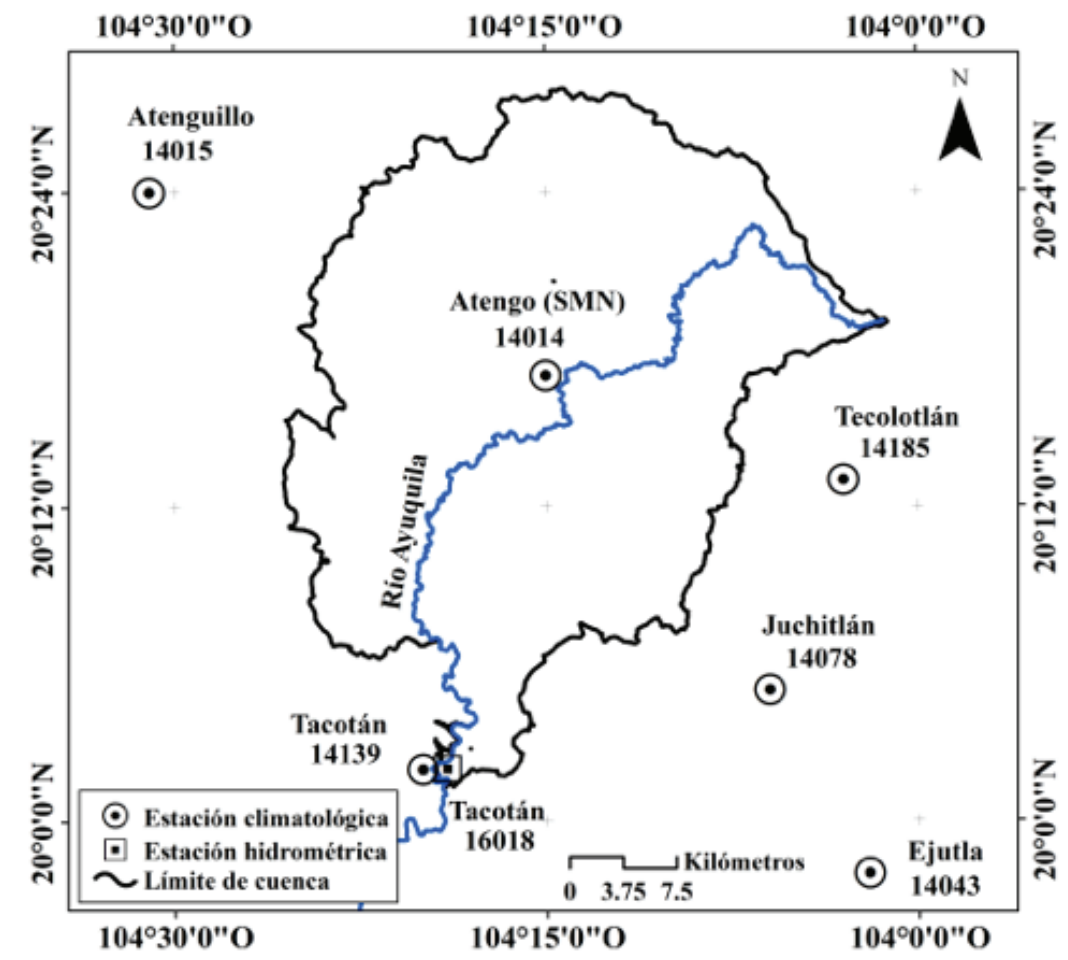

Figura 3. Localización de las estaciones climatológicas e hidrométrica, utilizadas para la etapa de calibración en la subcuenca Tacotán.

Cuadro 2. Factores utilizados para la determinación del coeficiente de escurrimiento en el Sistema de Información Geográfica (SIG).

\begin{tabular}{|c|c|c|c|}
\hline \multirow{2}{*}{ Topografía/uso de suelo } & \multicolumn{3}{|c|}{ Textura del suelo } \\
\hline & Franco arenoso (gruesa) & Arcilla y Franco-limoso (media) & Arcilla compactada (fina) \\
\hline Bosques & & & \\
\hline $0-5 \%$ pendiente & 0.10 & 0.30 & 0.40 \\
\hline $5-10 \%$ pendiente & 0.25 & 0.35 & 0.50 \\
\hline $1030 \%$ pendiente & 0.30 & 0.40 & 0.60 \\
\hline$>30 \%$ pendiente & 0.32 & 0.42 & 0.63 \\
\hline Pastizales & & & \\
\hline $0-5 \%$ pendiente & 0.15 & 0.35 & 0.45 \\
\hline $5-10 \%$ pendiente & 030 & 0.40 & 0.55 \\
\hline $1030 \%$ pendiente & 0.35 & 0.45 & 0.65 \\
\hline$>30 \%$ pendiente & 0.37 & 0.47 & 0.68 \\
\hline \multicolumn{4}{|l|}{ Terrenos agrícolas } \\
\hline $0-5 \%$ pendiente & 0.30 & 0.50 & 0.60 \\
\hline $5-10 \%$ pendiente & 0.40 & 0.66 & 0.70 \\
\hline $1030 \%$ pendiente & 0.50 & 0.70 & 0.80 \\
\hline$>30 \%$ pendiente & 0.53 & 0.74 & 0.84 \\
\hline
\end{tabular}


Cuadro 3. Factores utilizados para la estimación de la capacidad de campo en el Sistema de Información Geográfica (SIG).

\begin{tabular}{|c|c|c|}
\hline Uso de suelo y vegetación & Clase textural & $\mathrm{mm}$ \\
\hline \multirow{2}{*}{$\begin{array}{l}\text { Incluye asentamientos humanos, } \\
\text { infraestructura vial, sitios de extracción } \\
\text { de materiales, sin vegetación aparente }\end{array}$} & Media ( $35 \%$ arcilla y menos $65 \%$ de arena) & 5 (no hay suelo y solo se retiene en depresiones) \\
\hline & Fina (más del 35\% de arcilla) & 5 (no hay suelo y solo se retiene en depresiones) \\
\hline \multirow{2}{*}{$\begin{array}{l}\text { Cultivos de riego. Incluye áreas } \\
\text { inundables }\end{array}$} & Fina (más del 35\% de arcilla) & 100 \\
\hline & No especificado & 50 corresponde al embalse \\
\hline \multirow{3}{*}{ Cultivos de temporal } & Media (35\% arcilla y menos $65 \%$ de arena) & 200 \\
\hline & Fina (más del 35\% de arcilla) & 150 \\
\hline & No especificado & 50 corresponde al embalse \\
\hline \multirow{3}{*}{ Pastizales y matorrales (secundarios) } & Media (35\% arcilla y menos $65 \%$ de arena) & 150 \\
\hline & Fina (más del 35\% de arcilla) & 200 \\
\hline & No especificado & 75 \\
\hline \multirow{2}{*}{$\begin{array}{l}\text { Vegetación natural perturbada } \\
\text { (matorral xerófilo crassicaule, selva } \\
\text { baja caducifolia, bosque de encino y } \\
\text { vegetación riparia) }\end{array}$} & Media ( $35 \%$ arcilla y menos $65 \%$ de arena) & 300 \\
\hline & Fina (más del 35\% de arcilla) & 250 \\
\hline $\begin{array}{l}\text { Vegetación natural conservada (selva } \\
\text { baja caducifolia, matorral xerófilo }\end{array}$ & Media ( $35 \%$ arcilla y menos $65 \%$ de arena) & 350 \\
\hline $\begin{array}{l}\text { crassicaule, bosque de encino y } \\
\text { vegetación mixta). Incluye cauces }\end{array}$ & Fina (más del 35\% de arcilla) & 300 \\
\hline Cuerpo de agua & No especificado & 60 \\
\hline
\end{tabular}

\section{Coeficiente de infiltración $\left(C_{i}\right)$}

Es el coeficiente que más influye en la infiltración de la lluvia en el suelo, además de la textura del suelo, existen factores como la pendiente del terreno y la vegetación que son importantes en la infiltración (ONU, 1972; Schosinsky y Losilla, 2000). Este coeficiente de infiltración $\left(\mathrm{C}_{\mathrm{i}}\right)$, viene a conformar la infiltración máxima $\left(\mathrm{I}_{\max }\right)$ dentro del modelo de Témez. Basándonos en la información del "Manual de Instrucciones de Estudios Hidrológicos" realizado por las Naciones Unidas (1972) y los trabajos expuestos por Schosinsky y Losilla (2000), se propone $\mathrm{C}_{\mathrm{i}}$ definidos en el Cuadro 4.

La ecuación para el cálculo del coeficiente de infiltración se expresa en la siguiente fórmula:

$\mathrm{C}_{\mathrm{i}}=\mathrm{K}_{\mathrm{fc}}+\mathrm{K}_{\mathrm{p}}+\mathrm{K}_{\mathrm{v}}$

donde: $\mathrm{C}_{\mathrm{i}}=$ coeficiente de infiltración igual o menor a uno; $\mathrm{K}_{\mathrm{fc}}=$ fracción que infiltra por efecto de textura del suelo; $K_{p}=$ fracción que infiltra por efecto de pendiente; $\mathrm{K}_{\mathrm{v}}=$ fracción que infiltra por efecto de cobertura vegetal. $\mathrm{Si} \mathrm{K}_{\mathrm{fc}}+\mathrm{K}_{\mathrm{p}}+\mathrm{K}_{\mathrm{v}}$ es mayor de 1, $\mathrm{C}_{\mathrm{i}}=1$. Si $P<5 \mathrm{~mm} \mathrm{mes}^{-1}, \mathrm{C}_{\mathrm{i}}=0$.

El coeficiente de infiltración, es adimensional y viene a complementar la infiltración máxima $\left(\mathrm{I}_{\max }\right)$, se estima que, en cada precipitación, el follaje intercepta un porcentaje de humedad, en nuestro caso, asumiremos una retención del $12 \%$ para toda la cuenca, parecido a los estudios establecidos por Schosinsky y Losilla (2000), siguiendo la fórmula;

$\mathrm{I}_{\max }=\left(1-\mathrm{K}_{\mathrm{i}}\right) \mathrm{C}_{\mathrm{i}} \mathrm{P}$

donde: $\mathrm{I}_{\max }=$ infiltración máxima; $\mathrm{P}=$ precipitación mensual (mm); $C_{i}=$ coeficiente de infiltración igual o menor a uno; $\mathrm{K}_{\mathrm{i}}=0.12$, fracción interceptada por el follaje.

\section{Etapa de Calibración}

En esta etapa se estima con mayor precisión la relación de los caudales, usando los datos del caudal 
Cuadro 4. Factores utilizados para la determinación del coeficiente de infiltración en el Sistema de Información Geográfica (SIG).

\begin{tabular}{|c|c|c|c|c|c|}
\hline Textura de suelos & $\begin{array}{c}\text { Kfc } \\
(0.01 \%)\end{array}$ & Pendiente & $\begin{array}{c}\mathrm{Kp} \\
(0.01 \%)\end{array}$ & Cobertura Vegetal & $\begin{array}{c}\mathrm{Kv} \\
(0.01 \%)\end{array}$ \\
\hline Arcilla compactada impermeable (A) & 0.10 & $\begin{array}{l}\text { Muy plana } \\
(0-0.03 \%)\end{array}$ & 0.30 & Coberturas con zacate $<50 \%$ & 0.09 \\
\hline Combinación de limo y arcilla (B) & 0.20 & $\begin{array}{c}\text { Plana } \\
(0.3-1 \%)\end{array}$ & 0.15 & Terrenos cultivados & 0.10 \\
\hline \multirow[t]{3}{*}{ Suelos limoarenoso no muy compactados (C) } & 0.40 & $\begin{array}{c}\text { Algo plana } \\
(1-2 \%)\end{array}$ & 0.10 & Coberturas con pastizales & 0.18 \\
\hline & & $\begin{array}{l}\text { Promedio } \\
\quad(2-75)\end{array}$ & 0.05 & Bosques & 0.20 \\
\hline & & $\begin{array}{l}\text { Fuerte } \\
(>7 \%)\end{array}$ & 0.06 & Coberturas con zacate más de $75 \%$ & 0.21 \\
\hline
\end{tabular}

calculado y observado de 1943-1962, se eligió la combinación para la cual la correlación entre los valores de las variables de salida generadas por el modelo y las observadas en la estación hidrométrica sean coincidentes (Ponce, 1989). Para coincidir con la forma de los hidrogramas de los caudales calculados con los observados, se ajustaron los parámetros, $\alpha$ y $\beta$ que forman parte del factor de agotamiento del acuífero, el ajuste de estos parámetros representó una mejor respuesta hidrológica (Jiménez et al., 2002).

Los parámetros $\alpha$ y $\beta$, informan sobre la escorrentía subterránea, si las aportaciones en los meses de estiaje son muy similares, el valor de $\beta$ es alto, significa que la cuenca tiene una elevada regulación natural. Al contrario, en escasez de lluvias si las aportaciones difieren entre sí, el valor de $\beta$ será muy bajo, lo que indica que la cuenca está escasamente regulada por los acuíferos (Murillo y Navarro, 2011).

\section{Etapa de Verificación}

Se utilizaron, años diferentes a la calibración (19631978), se extrapolaron los valores ajustados de los parámetros $\alpha$ y $\beta$, utilizados en la etapa de calibración, con esto se mide el grado de predicción de nuestra calibración (Ponce, 1989). Para evaluar el grado de ajuste de los valores calculados con los observados, se utilizaron los modelos estadísticos de Nash-Sutcliffe Efficiency (NSE), Coeficiente de Correlación $\left(\mathrm{R}^{2}\right)$ y Coeficiente de Determinación ( $\mathrm{r}$ ). Validados los resultados en la etapa de calibración y la verificación, el modelo matemático de Témez está listo para ser utilizado en la etapa de predicción de la modelización de la cuenca del Río Ayuquila-Armería.

\section{Cuenca Ayuquila-Armería}

La cuenca se dividió en 96 subcuencas, con el fin de atenuar los efectos de la distribución de la lluvia y las temperaturas, se extrapolaron a cada una de las subcuencas los valores de los parámetros $\alpha$ y $\beta$, utilizados en la etapa de calibración, y verificación, se extrajeron el valor medio ponderado por superficie de los parámetros físicos y climáticos. Finalmente, las fórmulas expresadas en el modelo de Témez, se calcularon para cada una de las subcuencas, y la sumatoria resultó en el caudal total. Para verificar los regímenes reconstruidos se usó la información hidrométrica de los regímenes naturales observados de las estaciones: 11014 del Río Acaponeta en el estado de Nayarit, con fecha de 1946-2002; la estación 15005 del Río Cuitzmala en Jalisco, con fecha de 1965-1987, y la estación 16022 del Río Coahuayana en el estado de Colima, con fecha de 1949-1986. Para poder comparar los caudales calculados por el modelo y los observados por las estaciones hidrométricas, es necesario normalizar los resultados, haciendo un cociente entre los caudales medios mensuales (Q) sobre la superficie de la cuenca (A). El modelo de evaluación utilizado para medir el grado de ajuste, entre los valores calculados por el modelo de Témez versus observados por las estaciones hidrométricas, fue el coeficiente de correlación $\left(\mathrm{R}^{2}\right)$. 


\section{RESULTADOS Y DISCUSIÓN}

A partir de los datos sin calibrar obtenidos a través de las fórmulas expuestas por Témez de $\alpha 0.59$ y $\beta 0.96$, en el hidrograma de caudales, se observa que el modelo sobrestima el 50\% los caudales bajos para los 20 años, mientras que los caudales altos son sobrestimados en el 25\% y subestimados en el 35\% (Figura 4a). En resumen, los datos sin calibrar presentan un buen ajuste con los datos observados en el hidrograma.

Para la calibración, se ajustaron los parámetros, $\alpha 0.42$ y $\beta 0.80$, como resultado de este ajuste en el hidrograma los caudales bajos se asociaron mejor a lo largo del periodo observado de 1943-1962. Mientras tanto los caudales altos en el hidrograma sobrestimaron en un $40 \%$ y subestimaron en un $15 \%$ los caudales observados (Figura 4b). En síntesis, la calibración presentó un mejor ajuste de los caudales calculados con los observados, con respecto a los datos sin calibrar (Cuadro 5).

El resultado de la verificación del modelo, utiliza el ajuste de $\alpha$ y $\beta$ en la etapa de calibración (Cuadro 5), en el hidrograma se observó que el modelo de predicción, mantiene un buen ajuste en años diferentes a la etapa de calibración, entre los caudales bajos calculados con relación a los observados (Figura 4c). Los caudales altos en el modelo sobrestiman y subestiman el $18 \%$
Cuadro 5. Resultados de los modelos de evaluación.

\begin{tabular}{lccc}
\hline & No calibrados & Calibrados & Verificados \\
\hline Años registrados & 20 & 20 & 16 \\
Datos & 240 & 240 & 192 \\
$\begin{array}{l}\text { Eficiencia Nash- } \\
\begin{array}{l}\text { Sutcliffe Efficiency } \\
\text { (NSE) }\end{array}\end{array}$ & 0.63 & 0.70 & 0.65 \\
$\begin{array}{l}\text { Coeficiente de } \\
\text { determinación }\left(\mathrm{R}^{2}\right)\end{array}$ & 0.67 & 0.70 & 0.65 \\
$\begin{array}{l}\text { Coeficiente de } \\
\text { correlación } \mathrm{r} \\
(P<0.01)\end{array}$ & 0.82 & 0.83 & 0.81 \\
\hline
\end{tabular}

de los caudales observados, en general se observó una buena asociación entre los caudales calculados y observados.

Al ajustar $\alpha$ y $\beta$, se describe de mejor forma el comportamiento de los caudales calculados con los observados, mejorando el resultado de los modelos de evaluación, en los tres casos, los resultados fueron aceptables para NSE $>0.63,>0.70 \mathrm{y}>0.65$, para $\mathrm{R}^{2}>0.67,>0.70 \mathrm{y}>0.65$ (Cuadro 5). En el análisis de regresión, los valores obtenidos fueron altamente significativos, con una probabilidad de $(P<0.01)$. Se aprecia que los modelos de evaluación explican por
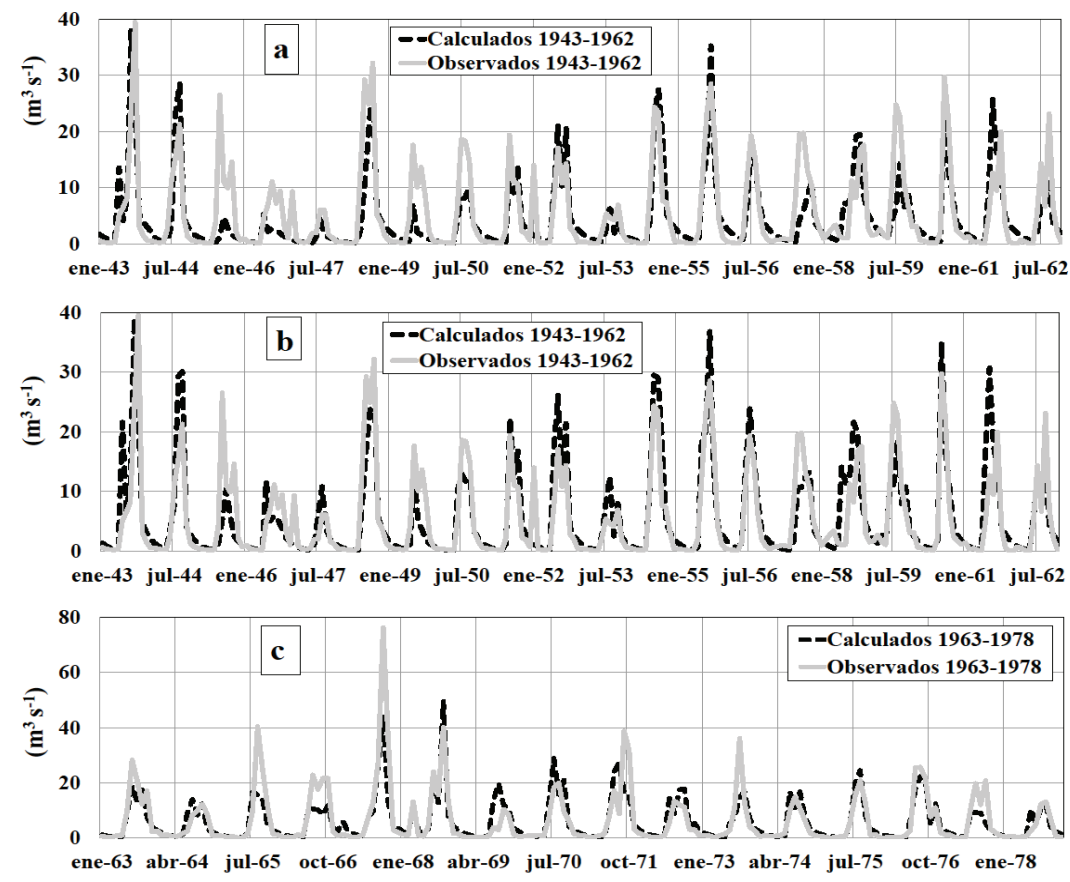

Figura 4. Hidrogramas de relación de caudales, (a) sin calibrar, (b) calibrados, y (c) verificados. Validación del modelo de Témez (1977). 
arriba del $80 \%$ de la variabilidad mensual para los tres casos (Cuadro 5), similar a los estudios de Pizarro et al. (2005), en dos cuencas de Chile central, donde los resultados obtenidos poseen la facultad de simular caudales a partir de datos climatológicos, completando series históricas en cuencas donde no se poseen datos hidrométricos.

Los resultados expuestos anteriormente para los modelos de evaluación: Nash-Sutcliffe Efficiency (NSE), coeficiente de determinación $\left(\mathrm{R}^{2}\right)$ y coeficiente de correlación (r), reflejaron un buen ajuste entre los caudales calculados por el modelo y los observados en la etapa de calibración y verificación, para la subcuenca Tacotán, lo que prueba la capacidad que tiene el modelo para predecir los valores promedios mensuales, con estos resultados estadísticos el modelo de Témez está listo para la etapa de simulación de toda la cuenca.

\section{Reconstrucción del Régimen Natural de Caudales}

El resultado es una base de datos del régimen natural de caudales para el periodo de 1963-1991 (Cuadro 6). En el análisis del hidrograma, se observó que el periodo intranual presentó marcadas las pautas estacionales, de febrero-mayo (estiaje), es alimentada por el caudal subterráneo (flujo base), las primeras lluvias no agregan caudal superficial, este se da una vez que, la capacidad de campo es superada, a finales de mayo o mediados

Cuadro 6. Resultado del régimen natural de caudales a la salida de la cuenca (1963-1991) $\left(\mathrm{m}^{3} \mathrm{~s}^{-1}\right)$.

\begin{tabular}{|c|c|c|c|c|c|c|c|c|c|c|c|c|}
\hline Años & Enero & Febrero & Marzo & Abril & Mayo & Junio & Julio & Agosto & Septiembre & Octubre & Noviembre & Diciembre \\
\hline 1963 & 21.5 & 11.63 & 10.62 & 5.53 & 4.38 & 75.98 & 186.73 & 141.56 & 186.83 & 103.97 & 34.21 & 45.28 \\
\hline 1964 & 21.63 & 14.19 & 9.31 & 6.11 & 4.01 & 71.42 & 133.53 & 100.89 & 167.17 & 38.33 & 22.94 & 15.12 \\
\hline 1965 & 9.91 & 6.51 & 4.27 & 2.8 & 1.86 & 11 & 127.69 & 178.6 & 151.4 & 48.08 & 26.84 & 25.75 \\
\hline 1966 & 14.78 & 9.73 & 6.38 & 4.19 & 2.77 & 70.9 & 117.78 & 158.24 & 198.13 & 172.04 & 43.09 & 28.28 \\
\hline 1967 & 105.29 & 26.68 & 17.51 & 11.49 & 7.58 & 36.62 & 93.6 & 216.08 & 525.92 & 172.08 & 55.34 & 36.73 \\
\hline 1968 & 24 & 16.66 & 95.39 & 22.39 & 15.07 & 36.68 & 220.25 & 105.58 & 459.93 & 75.48 & 39.31 & 29.34 \\
\hline 1969 & 18.49 & 12.13 & 7.96 & 5.22 & 3.43 & 8.36 & 130.15 & 188.36 & 198.1 & 181.29 & 38.47 & 25.32 \\
\hline 1970 & 16.61 & 10.9 & 7.15 & 4.69 & 3.08 & 132.25 & 250.25 & 166.91 & 281.21 & 55.7 & 32.27 & 21.17 \\
\hline 1971 & 13.89 & 9.11 & 5.98 & 3.92 & 2.57 & 79.44 & 240.65 & 338.75 & 310.67 & 202 & 51.39 & 33.59 \\
\hline 1972 & 22.04 & 14.46 & 9.49 & 6.23 & 4.3 & 118.59 & 157.54 & 207.62 & 237.4 & 62.52 & 97.51 & 34.87 \\
\hline 1973 & 23.41 & 15.25 & 10 & 6.56 & 4.32 & 41.72 & 117.49 & 243.57 & 273.62 & 152.96 & 43.86 & 28.73 \\
\hline 1974 & 18.85 & 12.37 & 8.12 & 5.33 & 10.86 & 140.51 & 206.81 & 185.91 & 152.54 & 45.21 & 28.44 & 19.19 \\
\hline 1975 & 12.53 & 8.22 & 5.39 & 3.54 & 2.32 & 67.16 & 299.67 & 356.26 & 167.12 & 65.04 & 32.76 & 21.49 \\
\hline 1976 & 14.1 & 9.25 & 6.07 & 3.98 & 2.61 & 80.46 & 346.85 & 288.94 & 199.14 & 67.39 & 205.64 & 43.62 \\
\hline 1977 & 28.6 & 18.77 & 12.31 & 8.08 & 5.3 & 103.98 & 190.75 & 158.82 & 139.13 & 51.62 & 28.91 & 18.76 \\
\hline 1978 & 12.31 & 8.07 & 5.3 & 3.48 & 2.29 & 109.38 & 142.07 & 175.13 & 282.4 & 87.8 & 36.11 & 23.69 \\
\hline 1979 & 15.55 & 10.2 & 6.69 & 4.39 & 2.88 & 23.24 & 290.6 & 177.83 & 122.92 & 33.98 & 21.24 & 13.94 \\
\hline 1980 & 40.83 & 12.67 & 8.31 & 5.46 & 3.62 & 39.58 & 112.33 & 168.39 & 199.63 & 68.46 & 32.83 & 22.08 \\
\hline 1981 & 52.57 & 19.12 & 12.53 & 8.22 & 5.39 & 37.2 & 202.71 & 173.05 & 206.38 & 58.66 & 37.99 & 21.95 \\
\hline 1982 & 14.41 & 11.33 & 6.65 & 4.36 & 2.86 & 11.03 & 165.92 & 154.09 & 101.61 & 36.18 & 63.87 & 26.35 \\
\hline 1983 & 17.31 & 11.17 & 7.33 & 4.81 & 16.06 & 14.03 & 145.42 & 171.38 & 197.71 & 53.72 & 32.16 & 19.64 \\
\hline 1984 & 13.09 & 8.57 & 5.63 & 3.69 & 2.51 & 82.22 & 182.37 & 169.09 & 211.1 & 52.43 & 29.31 & 19.23 \\
\hline 1985 & 12.62 & 8.28 & 5.43 & 3.56 & 2.34 & 149.18 & 232.14 & 172.64 & 160.61 & 77.14 & 32.34 & 21.2 \\
\hline 1986 & 13.91 & 9.13 & 5.99 & 3.93 & 2.58 & 66.65 & 184.69 & 127.89 & 221.27 & 154.34 & 41.88 & 26.97 \\
\hline 1987 & 17.69 & 11.73 & 7.69 & 5.05 & 3.31 & 72.71 & 309.99 & 148.75 & 126.49 & 36.79 & 23.63 & 15.5 \\
\hline 1988 & 10.17 & 6.67 & 4.45 & 2.91 & 1.91 & 110.82 & 181.37 & 218.21 & 285.33 & 57.96 & 29.56 & 19.39 \\
\hline 1989 & 12.72 & 8.35 & 5.48 & 3.59 & 2.36 & 13.25 & 116.45 & 144.87 & 118.95 & 49.33 & 22.84 & 16.05 \\
\hline 1990 & 10.33 & 6.79 & 4.45 & 2.92 & 1.92 & 57.76 & 140.4 & 190.55 & 228.11 & 73.34 & 33.22 & 21.8 \\
\hline 1991 & 14.3 & 9.38 & 6.16 & 4.04 & 2.65 & 77.79 & 227.14 & 129.95 & 129.29 & 41.02 & 24.48 & 15.85 \\
\hline
\end{tabular}


de junio. La época húmeda está marcada durante los meses de julio-octubre, alcanzando su máximo durante el mes de septiembre. Durante los meses de julioseptiembre, los caudales subterráneos en la cuenca son alimentados por la infiltración, en estos meses se da la recarga máxima en los mantos acuíferos. Al finalizar la época de lluvias, durante el mes de noviembre el flujo base es el que alimenta el caudal continuo dentro del río (Figura 5).

El resultado del balance del modelo de Témez, está formado por la entrada de lluvia que sale en forma de caudal, el análisis se realiza tomando en cuenta el año hidrológico de enero a diciembre. En la cuenca se estimó una precipitación de $836 \mathrm{~mm}$, de los cuales $593 \mathrm{~mm}$ formaron la evapotranspiración real y $243 \mathrm{~mm}$ el excedente, $195 \mathrm{~mm}$ fueron a dar al caudal superficial y $48 \mathrm{~mm}$ se infiltraron, éste coincide con el almacenamiento del acuífero de acuerdo con Témez (1977), del total infiltrado, solo $34 \mathrm{~mm}$ integraron el caudal base. La aportación total anual fue de $229 \mathrm{~mm}$, representando un caudal aproximado de $71.5\left(\mathrm{~m}^{3} \mathrm{~s}^{-1}\right)$ en la salida de la cuenca, y con un volumen aproximado anual de $2254\left(\mathrm{hm}^{3}\right.$ año $\left.^{-1}\right)$.

En la verificación del caudal reconstruido del Río Ayuquila-Armería, los resultados de los hidrogramas, se ajustan a los caudales bajos observados para la época de estiaje, para los Ríos Acaponeta, Cuitzmala y Coahuayana. En la época de lluvias de junio a septiembre, únicamente el Río Cuitzmala presentó diferencias muy marcadas, relacionándolo con el tamaño de la cuenca y el tiempo de concentración
(Figura 6a). En términos generales las correlaciones fueron positivas para los tres ríos con $\mathrm{R}^{2}>0.91$, $\mathrm{R}^{2}>0.87$ y $\mathrm{R}^{2}>0.76$ (Figura $6 \mathrm{~b}$ ). Con esto se prueba la capacidad que tiene el modelo de Témez para predecir regímenes naturales de caudales a una escala mensual.

Otros resultados encontrados en este estudio, fueron los que correspondieron a la distribución espacial de los parámetros físicos: coeficiente de escorrentía (Ce), $\mathrm{Ce}>0.50$ corresponde a las áreas agrícolas y abiertas de la cuenca, $\mathrm{Ce}<0.40$ son característicos de áreas forestales, como los encontrados en la Sierra de San Carlos en el estado de Tamaulipas por Treviño et al. (2002) (Figura 7a).

Para la capacidad de campo $\left(\mathrm{H}_{\max }\right)$, se identificaron áreas con mayor retención de humedad $>300 \mathrm{~mm}$, localizadas en las zonas forestales de la cuenca, en la meseta de Cerro Grande dentro la Reserva de la Biosfera Sierra de Manantlán (RBSM), las faldas del Nevado de Colima, las Sierras de Cacoma, Tapalpa y Quila. Las menores retenciones de humedad se localizan en los sitios agrícolas de la cuenca (Figura 7b).

Las áreas de mayor infiltración, se localizaron en el valle Grullo-Autlán y Unión de Tula. El domo kárstico de Cerro Grande dentro la RBSM presentó valores entre 0.30 a 0.70 del total anual precipitado, estos valores se asemejaron a los encontrados por Espíritu (2011) en Cerro Hueco en Tuxtla Gutiérrez en el estado de Chiapas, y Kessler (1965) para las regiones kársticas de Hungría. Las cubiertas forestales de toda la cuenca presentaron valores de 0.30 a 0.50 estas áreas son importantes para el mantenimiento de un caudal continuo (Figura 7c).

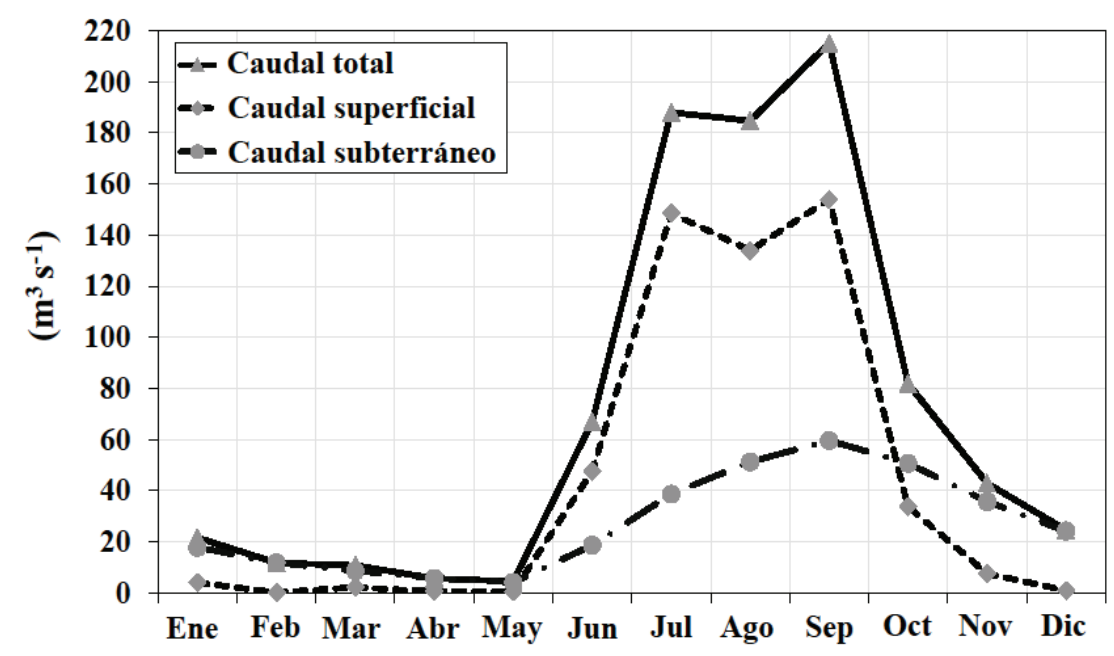

Figura 5. El hidrograma promedio de distribución mensual de 1963-1991. 

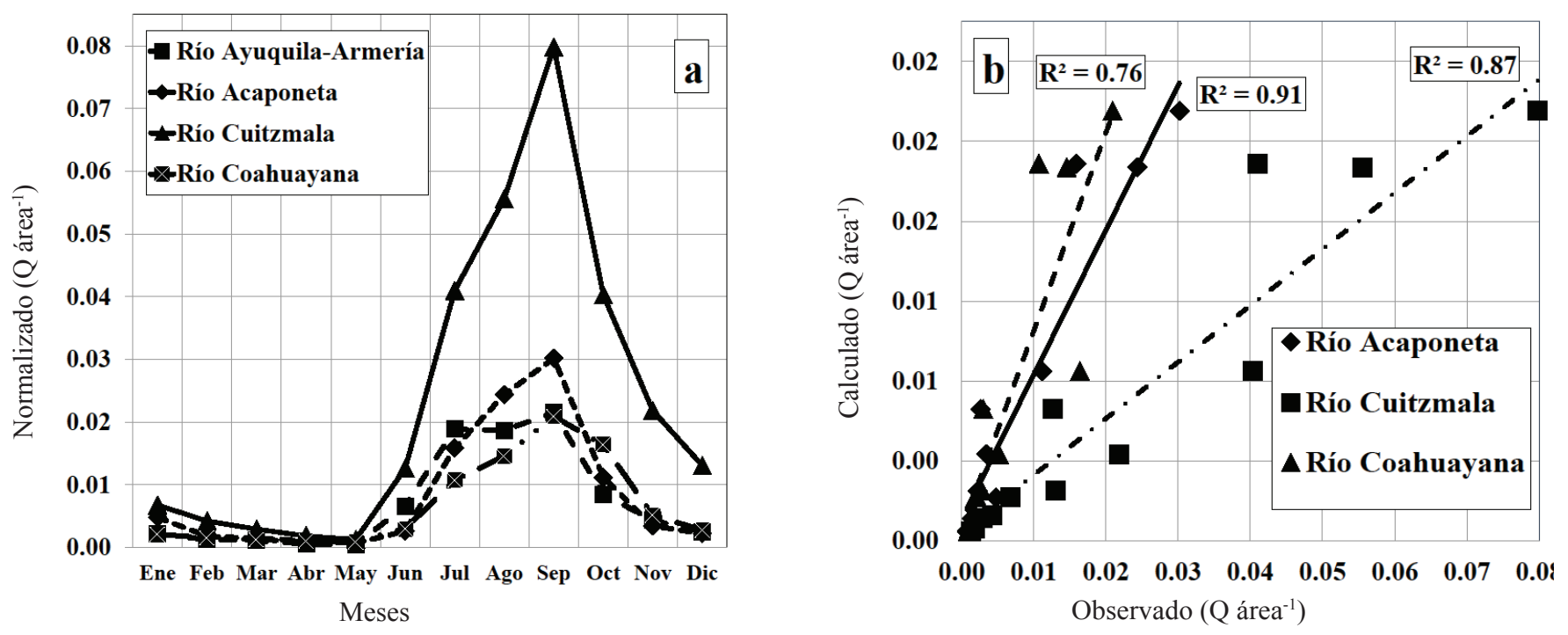

Figura 6. Hidrograma de caudales normalizados (a); modelo de correlación de caudales (b).
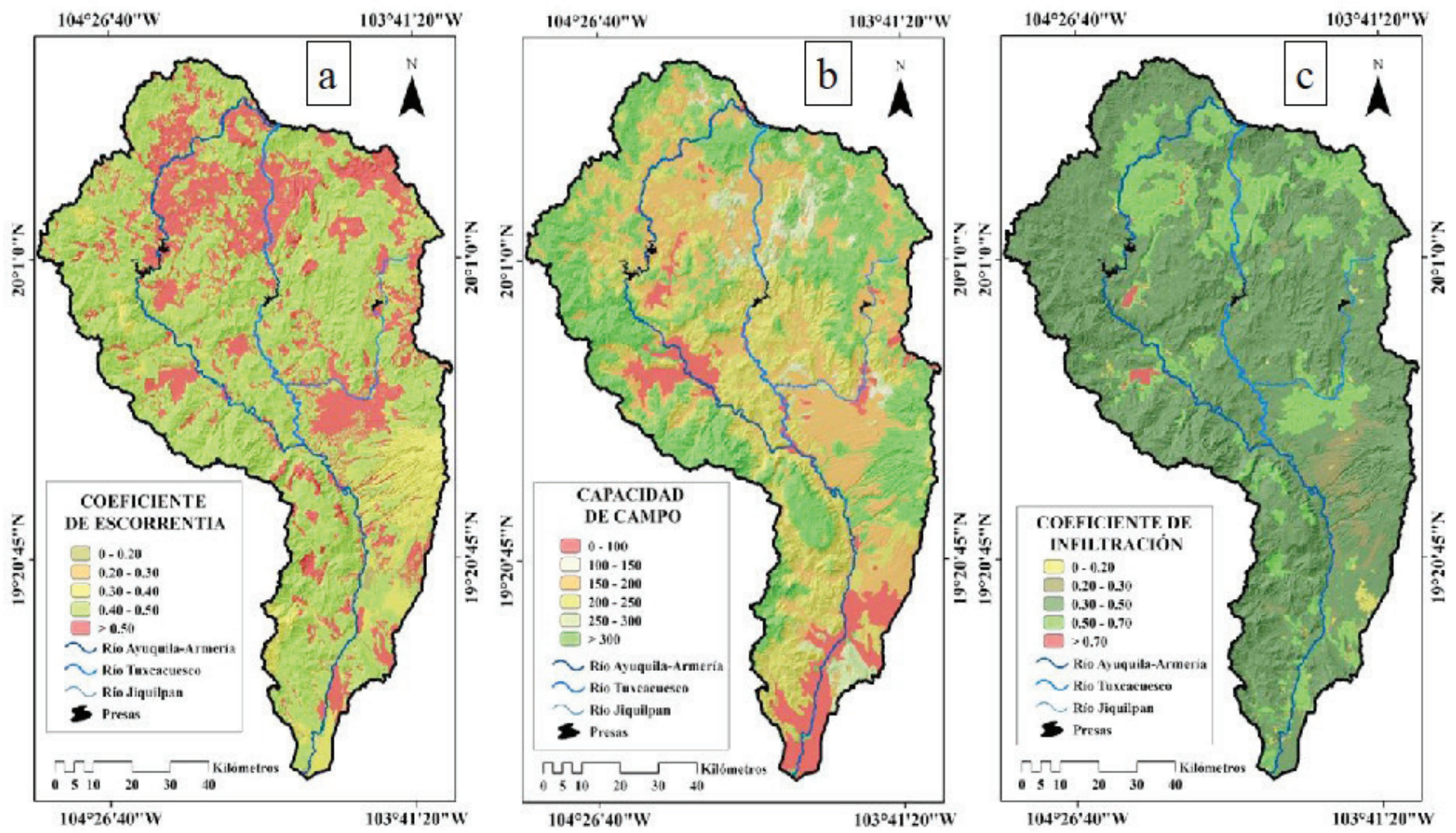

Figura 7. Mapa de valores indirectos, (a) coeficientes de escorrentía, (b) capacidad de campo y, (c) coeficiente de infiltración. 


\section{CONCLUSIONES}

El presente trabajo es una aproximación para la interpretación del caudal, superficial y subterráneo, en el Río Ayuquila-Armería y sus 96 subcuencas tributarias a una escala mensual. Este modelo de simulación continua tiene la capacidad de estimar series históricas mensuales de caudales naturales a partir de datos meteorológicos, en cuencas donde se carece de información hidrométrica puntual. Su aplicación, se convierte en una herramienta para la planificación y diseño de las obras hidráulicas, además de permitir entender el significado ecológico de cada componente del régimen hidrológico natural para generar propuestas para su conservación como lo pide la norma NMX-AA-159-SCFI-2012 que establece el procedimiento para la determinación del caudal ecológico en México. Entre otras cosas estos resultados permitirán identificar las alteraciones hidrológicas mediante el análisis comparativo de estas series, la aplicación de esta técnica puede ser extendida a otras cuencas en México y Latinoamérica, donde se carece de datos hidrométricos puntuales.

\section{LITERATURA CITADA}

Barrios, A. G. y L. A. Urribarri. 2010. Aplicación del modelo Swat en los Andes venezolanos: Cuenca alta del río Chama. Rev. Geogr. Venez. 51: 11-29.

Estrela, M. T. 1992. Modelos matemáticos para la evaluación de recursos hídricos. Centro de Estudios y Experimentación de Obras Públicas. CEDEX. Madrid, España.

Espíritu, T. G. 2011. Segundo Congreso Nacional de Manejo de Cuencas Hidrográficas. Ponencia titulada "Cerro Hueco y su Agua Subterránea, una Microcuenca Conurbana a Tuxtla Gutiérrez, Chiapas" Universidad Juárez Autónoma de Tabasco. Villahermosa, Tabasco. México. http://www.inecc.gob.mx/ descargas/cuencas/congnal_06/tema_05/08_gloria_ espíritu 1.pdf (Consulta: agosto 16, 2013).

FAO (Food and Agriculture Organization of the United Nations). 2015. World reference base for soil resources 2014. International soil classification system for naming soils and creating legends for soil maps. Food and Agriculture Organization of the United Nations. Rome, Italy.

Flores-López, H. E., H. Ramírez-Vega, K. F. Byerly-Murphy, J. A. Ruiz-Corral, J. A. Martínez-Sifuentes, P. Díaz-Mederos y V. Alemán-Martínez. 2003. Estimación de escurrimiento superficial en la cuenca El Jihuite, México. Terra Latinoamericana 21: 389-400.

Horn, B. K. P. 1981. Hill shading and the reflectance map. Proc. IEEE 69: 14-47.
INEGI (Instituto Nacional de Estadística y Geografía) 2013. Conjunto de datos vectoriales de uso del suelo y vegetación. Escala 1:250 000, Serie V (Capa Unión). Aguascalientes, México. http://www.inegi.org.mx/geo/contenidos /recnat/ usosuelo/Default.aspx. (Consulta: junio 24, 2015).

INEGI (Instituto Nacional de Estadística y Geografía). 2013. Conjunto de datos vectorial edafológica escala 1:250 000, Serie II (Continuo Nacional). Aguascalientes, México. http://www.inegi.org.mx/geo/contenidos/recnat/edafologia/ vectorial_serieii.aspx. (Consulta: octubre 22, 2015).

INEGI (Instituto Nacional de Estadística y Geografía). 2015. Conjunto de datos espaciales: Información topográfica a escala 1:50,000. Aguascalientes, México. http://www.inegi. org. $\mathrm{mx} /$ sistemas $/$ biblioteca $/$ detalle2 .aspx $? \mathrm{c}=2354 \&$ upc $=0 \& \mathrm{~s}$ $=$ geo $\& \operatorname{tg}=999 \& \mathrm{f}=2 \& \mathrm{cl}=0 \& \mathrm{pf}=$ Prod $\& \mathrm{ef}=0 \& \mathrm{ct}=206000000$ (Consulta: agosto 16, 2015).

CONAGUA-SEMARNAT-IMTA (Comisión Nacional de AguaSecretaría de Medio Ambiente y Recursos Naturales-Instituto Mexicano de Tecnología del Agua). 2008. Banco Nacional de Datos de Aguas Superficiales (BANDAS), 8 CD's. Jiutepec, Morelos. México.

Jiménez, G. P., B. A. Navarro, F. C. Carrasco Cantos, J. J. V. Durán, J. A. G. López e I. P. Vadillo. 2002. Aplicación de un modelo lluvia-caudal al acuífero carbonático de la Sierra de las Cabras (Provincia de Cádiz, S de España). Geogaceta: 31: 63-66.

Kessler, H. 1965. Water balance investigations in the Karstic regions of Hungary. Symposium on hydrology of fractured rocks. IHD: International Hydrological Decade, 1965-1975. Dubrovnik, Yugoslavia.

ONU (Organización de la Naciones Unidades). 1972. Manual de instrucciones, estudios hidrológicos. Organización de la Naciones Unidades. Publicación número 70. San José, Costa Rica.

Murillo D., J. M. y J. A. Navarro. 2011. Aplicación del modelo de Témez a la determinación de la aportación superficial y subterránea del sistema hidrológico Cornisa-Vega de Granada para su implementación en un modelo de uso conjunto. Bol. Geol. Min. 122: 363-388.

Norma Oficial Mexicana NMX-AA-159-SCFI. 2012. Que establece el procedimiento para la determinación del caudal ecológico en cuencas hidrológicas. Diario Oficial de la Federación. México, D. F.

Norma Oficial Mexicana NOM-011-CONAGUA. 2015. Conservación del recurso agua-Que establece las especificaciones y el método para determinar la disponibilidad media anual de las aguas nacionales. Diario Oficial de la Federación. México, D. F.

Ortiz, V. B. y C. A. Ortiz. 1990. Edafología. Universidad Autónoma Chapingo. Departamento de Suelos. Chapingo, México.

Pizarro T., R., M. Soto B., C. Farías D. y C. Jordán D. 2005. Aplicación de dos modelos de simulación integral hidrológica, para la estimación de caudales medios mensuales, en dos cuencas de Chile central. Bosque 26: 123-129.

Ponce M. V. 1989. Catchment Modeling. pp. 400-410. I: V. M. Ponce (ed.). Engineering hydrology: Principles and practices. Prentice Hall. UK. 
SMN (Servicio Meteorológico Nacional). 2014. Bases de datos meteorológicos del sistema de clima computarizado (CLICOM). Disco compacto. México, DF.

Schosinsky, G. y M. Losilla. 2000. Modelo analítico para determinar la infiltración con base en la lluvia mensual. Rev. Geol. Am. Cent. 23: 43-55.

Schloeder, C. A., N. E. Zimmerman, and M. J. Jacobs. 2001. Comparison of methods for interpolating soil properties using limited data. Soil Sci. Soc. Am. J. 65: 470-479.

Témez, P. J. 1977. Modelo Matemático de transformación "precipitación-aportación". Asociación de Investigación
Industrial Eléctrica. Comisión E. <Explotación y Garantía $>$ Grupo de Trabajo de Predicciones de Precipitación y Relación entre Precipitaciones y Caudales. Madrid, España.

Thornthwaite, C. W. and J. R. Mather. 1957. Instructions and tables for computing potential evapotranspiration and the water balance. Publications in Climatology. Drexel Institute of Technology. Laboratory of Climatology. Centerton, NJ, USA.

Treviño G., E. J., C. A. Muñoz R., C. Cavazos C. y L. Barajas Ch. 2002. Evaluación del flujo hídrico superficial en la Sierra de San Carlos, Tamaulipas. Ciencia UANL 5: 525-530. 